PROCEEDINGS OF THE

AMERICAN MATHEMATICAL SOCIETY

Volume 125, Number 8, August 1997, Pages 2503-2510

S 0002-9939(97)03998-1

\title{
HOMOGENEITY IN POWERS OF ZERO-DIMENSIONAL FIRST-COUNTABLE SPACES
}

\author{
ALAN DOW AND ELLIOTT PEARL
}

(Communicated by Franklin D. Tall)

\begin{abstract}
A construction of L. Brian Lawrence is extended to show that the $\omega$-power of every subset of the Cantor set is homogeneous via a continuous translation modulo a dense set. It follows that every zero-dimensional firstcountable space has a homogeneous $\omega$-power.
\end{abstract}

\section{INTRODUCTION}

A topological space is homogeneous if for any pair of points of the space there is an autohomeomorphism of the space mapping one point to the other. Nonhomogeneous spaces may have a homogeneous power. O.H. Keller [3] showed that the Hilbert cube $[0,1]^{\omega}$ is homogeneous. D.B. Motorov [6] proved that if $X$, a zero-dimensional first-countable space, has a dense set of isolated points then $X^{\omega}$ is homogeneous. A space is zero-dimensional if it has a $T_{1}$ basis of clopen sets.

G. Gruenhage [1] asked if $X^{\omega}$ is homogeneous for every zero-dimensional firstcountable space $X$. This question was also asked by H.-X. Zhou [8]. S.V. Medvedev [5] announced that if $X$ is a zero-dimensional metric space that is either first category in itself, or contains a dense complete subspace, then $X^{\omega}$ is homogeneous. F. van Engelen [7] independently obtained results similar to Medvedev's. G. Gruenhage and H.-X. Zhou [2] had shown already that if $X$ is a zero-dimensional first-countable space that either has a dense set of isolated points or is an absolutely Borel separable metric space then $X^{\omega}$ is homogeneous. Recently, L. Brian Lawrence [4] proved that if $X$ is a zero-dimensional subspace of the real line then $X^{\omega}$ is homogeneous. We use his technique to answer Gruenhage's question in the affirmative.

\section{LAWRENCE'S THEOREM}

Lawrence proved the following theorem in the context of $D \subset \omega^{\omega}$ and $X=\bar{D}$ not being compact. Lawrence did not need to prove this when $X$ is compact since in such a case $X^{\omega}$ is known to be homeomorphic to the Cantor set, hence homogeneous too. However, we will need the full strength of this result.

Received by the editors October 23, 1995 and, in revised form, March 4, 1996.

1991 Mathematics Subject Classification. Primary 54B10.

Key words and phrases. Homogeneous, zero-dimensional, first-countable, elementary submodel.

The first author acknowledges support from NSERC of Canada.

(C)1997 American Mathematical Society 
Theorem 1. If $D \subset 2^{\omega}, x, y \in D^{\omega}$, and $X=\bar{D}$ then there are an autohomeomorphism $f$ of $X^{\omega}$ and a map $h: z \mapsto h_{z}$ from $X^{\omega}$ to the set of partial permutations of $\omega$ satisfying

1. $f(x)=y$,

2. $\left(\forall z \in X^{\omega}\right)\left(\forall n \in \operatorname{dom} h_{z}\right)\left(\exists U n b^{\prime} h d\right.$ of $\left.z\right)(\forall w \in U) h_{w}(n)=h_{z}(n)$,

3. $\left(\forall z \in X^{\omega}\right) \begin{cases}f(z)(n)=z\left(h_{z}(n)\right), & n \in \operatorname{dom} h_{z}, \\ f(z(n)) \in D, & n \notin \operatorname{dom} h_{z}, \\ z(m) \in D, & m \notin \operatorname{ran} h_{z} .\end{cases}$

The homeomorphism $f$ is a continuous translation of $X^{\omega}$ modulo the dense set $D$. Pointwise, $f$ translates coordinates as coded by the partial permutation of $\omega$ given by $h$. The coordinates translated may vary from point to point, but they vary continuously. Not all coordinates necessarily get translated. However, coordinates that are not translated must have values in the dense set $D$. The intention is that $D$ should be a countable set, although this is not a necessary assumption. Observe that for all $A$ with $D \subset A \subset X, f\left(A^{\omega}\right)=A^{\omega}$.

Proof. Let $D \subset 2^{\omega}, x, y \in D^{\omega}$, and $X=\bar{D}$. Fix some well-ordering of $D$. Start with $k_{0}=\inf \left\{k \in \omega: \mid\{d\lceil k: d \in D\} \mid \geq 2\}, k_{1}=k_{0}+1\right.$, and define $k_{n+1}=k_{n-1}+k_{n}+2$ for $n \geq 1$. Identify $\left(2^{\omega}\right)^{\omega}=2^{\omega \times \omega}=2^{\omega^{2}}$ so that the following definitions make sense. Define $B_{n}=\left\{z\left\lceil k_{n}^{2}: z \in D^{\omega}\right\} . B=\bigcup\left\{B_{n}: n \in \omega\right\}\right.$ codes a clopen basis of $X^{\omega}$. Namely, for each $z \in D^{\omega}$ and $n \in \omega,\left[z\left\lceil k_{n}^{2}\right]=\left\{v \in X^{\omega}: z\left\lceil k_{n}^{2} \subset v\right\}\right.\right.$ is a basic clopen set. With its natural ordering of inclusion, $B$ is a tree. The plan is to construct a new tree ordering on $B$ that has the same levels as the inclusion tree and also agrees with the inclusion ordering for nodes that are an even number of levels apart. We will actually formally define the new tree by constructing its predecessor function $p$. It will then be possible to define an autohomeomorphism of $X^{\omega}$ by $f(z)=\bigcup\left\{p\left(z\left\lceil k_{2 n+1}^{2}\right): n \in \omega\right\}\right.$, for $z \in X^{\omega}$. We will also be approximating the coding map $h$ along the new tree. When $\sigma \in B_{n}, h_{\sigma}$, a partial permutation of $k_{n-1}$, codes the translation of coordinates between $p(\sigma)$ and $\sigma$. Eventually $h_{z}=\bigcup\left\{h_{z \nmid k_{2 n+1}^{2}}: n \in \omega\right\}$, for $z \in X^{\omega}$.

It will suffice for $p$ and $h$ to satisfy

1. $\left(\forall \sigma \in B_{n+1}\right) p(p(\sigma)) \subset \sigma$,

2. $\left(\forall \sigma \in B_{n}\right)\left(\forall i \in \operatorname{dom} h_{\sigma}\right)\left(\forall j \in k_{n-1}\right) p(\sigma)(i, j)=\sigma\left(h_{\sigma}(i), j\right)$,

3. $\left(\forall \sigma \in B_{n+1}\right) h_{\sigma} \neq h_{p(\sigma)}^{-1}$ implies $h_{\sigma}=h_{p(\sigma)}^{-1} \cup\left\{\left\langle\inf \left(\omega \backslash \operatorname{ran} h_{p(\sigma)}, k_{n-1}\right)\right\rangle\right\}$,

4. $h_{\sigma}=h_{p(\sigma)}^{-1}$ and $p(\sigma) \notin\left\{y\left\lceil k_{2 n}^{2}, x\left\lceil k_{2 n+1}^{2}: n \in \omega\right\}\right.\right.$ imply $h_{p(\sigma)} \neq h_{p(p(\sigma))}^{-1}$,

5. $\left(\forall \sigma \in B_{n+1}\right)\left(\forall i \in k_{n-1} \backslash \operatorname{ran} h_{\sigma}\right)\left(\forall j \in k_{n+1}\right) h_{\sigma} \neq h_{p(\sigma)}^{-1}$ implies

$$
\sigma(i, j)=\inf \left(D \cap\left[\left\langle\sigma(i, l): l \in k_{n-1}\right\rangle\right]\right)(j),
$$

6. $p\left(x\left\lceil k_{2 n+1}^{2}\right)=y\left\lceil k_{2 n}^{2}\right.\right.$ and $p\left(y\left\lceil k_{2 n+2}^{2}\right)=x\left\lceil k_{2 n+1}^{2}, h_{y \uparrow k_{2 n}^{2}}=\phi=h_{x \uparrow k_{2 n+1}^{2}}\right.\right.$.

$h$ is well defined as a union because condition 3 implies that $h_{\sigma} \supset h_{p(\sigma)}^{-1} \supset h_{p(p(\sigma))}$. Condition 1 ensures that $f$ is well defined via a union too. Condition 6 ensures that $f(x)=y$ along the distinguished $x-y$ branch and that $h_{x}$ is empty.

To see that $h$ satisfies the continuity property 2 of the theorem, let $z \in X^{\omega}$ and $n \in \operatorname{dom} h_{z}$. Find $l \in \omega$ such that $n \in \operatorname{dom} h_{z \nmid k_{2 l+1}^{2}} \subset \operatorname{dom} h_{z}$. Now $\left[z\left\lceil k_{2 l+1}^{2}\right]\right.$ is a neighbourhood of $z$ and if $w \in\left[z\left\lceil k_{2 l+1}^{2}\right]\right.$ then $h_{z \nmid k_{2 l+1}^{2}} \subset h_{w}$. In particular, $h_{w}(n)=h_{z \nmid k_{2 l+1}^{2}}(n)=h_{z}(n)$. 
Condition 2 ensures that the translation clause of property 3 of the theorem is satisfied. To see that the rest of property 3 is satisfied, let $z \in X^{\omega} \backslash\{x\}$ and $m \notin \operatorname{ran} h_{z}$. Claim

$$
(\exists L \in \omega)(\forall l \geq L) h_{z \mid k_{2 l+1}^{2}} \neq h_{p\left(z \nmid k_{2 l+1}^{2}\right)}^{-1} .
$$

Otherwise, there are infinitely many $l \in \omega$ such that $h_{z \uparrow k_{2 l+1}^{2}}=h_{p\left(z \nmid k_{2 l+1}^{2}\right.}^{-1}$. Condition 4 implies that when such $l \in \omega$ are large enough so that $p\left(z\left\lceil k_{2 l+1}^{2}\right) \neq y\left\lceil k_{2 l+1}^{2}\right.\right.$, $h_{p\left(z \nmid k_{2 l+1}^{2}\right)} \neq h_{p\left(p\left(z \nmid k_{2 l+1}^{2}\right)\right)}^{-1}$. Condition 3 implies that for such $l \in \omega$,

$$
h_{p\left(z \nmid k_{2 l+1}^{2}\right)}=h_{p\left(p\left(z \nmid k_{2 l+1}^{2}\right)\right)}^{-1} \cup\left\{\left\langle\inf \left(\omega \backslash \operatorname{ran} h_{p\left(p\left(z \nmid k_{2 l+1}^{2}\right)\right)}\right), k_{2 l-2}\right\rangle\right\} .
$$

Condition 1 ensures $p\left(p\left(z\left\lceil k_{2 l+1}^{2}\right)\right)=z\left\lceil k_{2 l-1}^{2}\right.\right.$. Thus, for infinitely many $l \in \omega$,

$$
\begin{aligned}
\inf \left(\omega \backslash \operatorname{ran} h_{z \nmid k_{2 l-1}^{2}}\right) & =\inf \left(\omega \backslash \operatorname{ran} h_{p\left(p\left(z \nmid k_{2 l+1}^{2}\right)\right)}\right) \\
& \in \operatorname{dom} h_{p\left(z \nmid k_{2 l+1}^{2}\right)} \\
& =\operatorname{ran} h_{p\left(z \nmid k_{2 l+1}^{2}\right)}^{-1} \\
& =\operatorname{ran} h_{z \nmid k_{2 l+1}^{2}} .
\end{aligned}
$$

But $h_{z}=\bigcup\left\{h_{z \nmid k_{2 l+1}^{2}}: l \in \omega\right\}$, therefore $\operatorname{ran} h_{z}=\omega$. This proves the claim. It may be assumed that $L$ is large enough so that $m \in k_{2 L-1}$. Claim $z(m) \in D$ because $z(m)=\inf \left(D \cap\left[z(m)\left\lceil k_{2 L-1}\right]\right) \in D\right.$. Actually, use induction on $l \geq L$ to show that

$$
(\forall l \geq L) z(m) \uparrow k_{2 l-1}=\inf \left(D \cap\left[z(m) \uparrow k_{2 L-1}\right]\right) \uparrow k_{2 l-1} .
$$

When $l \geq L, m \in k_{2 L-1} \backslash \operatorname{ran} h_{z} \subset k_{2 l-1} \backslash \operatorname{ran} h_{z \uparrow k_{2 l+1}^{2}}$. Now, the inductional step is verified via condition 5 , which says that

$$
\begin{aligned}
z(m) \uparrow k_{2 l+1} & =\inf \left(D \cap\left[\left\langle p\left(p\left(z\left\lceil k_{2 l+1}^{2}\right)\right)(m, s): s \in k_{2 l-1}\right\rangle\right]\right) \uparrow k_{2 l+1}\right. \\
& =\inf \left(D \cap\left[\left\langle\left(z\left\lceil k_{2 l-1}^{2}\right)(m, s): s \in k_{2 l-1}\right\rangle\right]\right) \uparrow k_{2 l+1}\right. \\
& =\inf \left(D \cap [ z ( m ) \lceil k _ { 2 l - 1 } ] ) \left\lceilk_{2 l+1}\right.\right. \\
& =\inf \left(D \cap\left[\inf \left(D \cap\left[z(m)\left\lceil k_{2 L-1}\right]\right) \uparrow k_{2 l-1}\right]\right) \uparrow k_{2 l+1}\right. \\
& =\inf \left(D \cap [ z ( m ) \lceil k _ { 2 L - 1 } ] ) \left\lceilk_{2 l+1} .\right.\right.
\end{aligned}
$$

Similarly, $f(z(n)) \in D$ when $n \notin \operatorname{dom} h_{z}$.

Towards defining $p$ and $h$, we will also define a function $\theta$, that will describe a good candidate for a new node to put between two nodes that are two levels apart and comparable in the inclusion order. The domain of $\theta$ will be all pairs $\langle\sigma, \tau\rangle \in B_{n} \times B_{n+2}$ with $\sigma \subset \tau$ and $\tau$ not on the $x-y$ branch. We will refer to this set as $\operatorname{dom} \theta$ even though $\theta$ has not yet been defined. We will ensure that $\theta(\sigma, \tau)$ is one-to-one with respect to $\sigma$ and does not lie on the $x-y$ branch.

To establish the distinguished $x-y$ branch we now define $p\left(x \nmid k_{2 n+1}^{2}\right)=y\left\lceil k_{2 n}^{2}\right.$, $p\left(y\left\lceil k_{2 n+2}^{2}\right)=x\left\lceil k_{2 n+1}^{2}\right.\right.$. Also define $h_{y\left\lceil k_{2 n}^{2}\right.}=\phi=h_{x \uparrow k_{2 n+1}^{2}}$ for all $n \in \omega$.

Starting at $B_{0}$, we do not need to define $p(\sigma)$ or $h_{\sigma}$. For $\langle\sigma, \tau\rangle \in \operatorname{dom} \theta$ with $\sigma \in B_{0}$, define $\theta(\sigma, \tau) \in B_{1}$ for $i, j \in k_{1}$ by

$$
\theta(\sigma, \tau)(i, j)= \begin{cases}\tau(i, j), & i \in k_{0}, \\ \inf \left(D \backslash\left[y\left(k_{0}\right)\left\lceil k_{0}\right]\right)(j),\right. & i=k_{0} .\end{cases}
$$

Next, for $\sigma \in B_{1} \backslash\left\{y\left\lceil k_{1}^{2}\right\}\right.$, define $p(\sigma)=\sigma \uparrow k_{0}^{2}$ and $h_{\sigma}=i d_{k_{0}}$. 
Suppose $p(\sigma), h_{\sigma}$, and $\theta\left(\sigma^{\prime}, \tau^{\prime}\right)$ have been defined for $\sigma \in B_{n},\left\langle\sigma^{\prime}, \tau^{\prime}\right\rangle \in \operatorname{dom} \theta$ with $\sigma^{\prime} \in B_{n-1}$, up to some $n \geq 1$. For $\langle\sigma, \tau\rangle \in \operatorname{dom} \theta$ with $\sigma \in B_{n}$, define $\theta(\sigma, \tau) \in B_{n+1}$ for $i, j \in k_{n+1}$ by

$$
\theta(\sigma, \tau)(i, j)= \begin{cases}\tau\left(h_{\sigma}(i), j\right), & i \in \operatorname{dom} h_{\sigma}, \\ d_{i}^{\sigma}(j), & i \in k_{n-1} \backslash \operatorname{dom} h_{\sigma}, \\ \tau\left(\inf \left(\omega \backslash \operatorname{ran} h_{\sigma}\right), j\right), & i=k_{n-1} \\ e_{i}^{n}(j), & i=k_{n-1}+1 \\ \tau\left(i-\left(k_{n-1}+2\right), j\right), & i \in k_{n+1} \backslash k_{n-1}+2,\end{cases}
$$

where

$$
\begin{aligned}
d_{i}^{\sigma} & =\inf \left(D \cap\left[\left\langle p(\sigma)(i, l): l \in k_{n-1}\right\rangle\right]\right), \\
e_{i}^{n} & = \begin{cases}\inf \left(D \backslash\left[x(i) \uparrow k_{0}\right]\right), & n \text { even, } \\
\inf \left(D \backslash\left[y(i) \uparrow k_{0}\right]\right), & n \text { odd. }\end{cases}
\end{aligned}
$$

Observe that $\theta(\sigma, \tau) \uparrow\left(k_{n+1} \backslash k_{n-1}+2\right) \times k_{n}$ is a copy of $\sigma$, independent of $h_{\sigma}$, therefore the function $\theta$ is one-to-one with respect to $\sigma$. The $e_{i}^{n}$ ensures that $\theta(\sigma, \tau)$ is not on the $x-y$ branch.

It remains to define $p(\sigma), h_{\sigma}$ for $\sigma \in B_{n+1}$ but not on the $x-y$ branch. This will depend on whether $\sigma \in \operatorname{ran} \theta$, that is, whether $\sigma$ was selected by $\theta$ as a good candidate to put between two nodes. When $\sigma \in \operatorname{ran} \theta$, there is a unique $\sigma^{\prime} \in B_{n}$ such that $\sigma=\theta\left(\sigma^{\prime}, \tau^{\prime}\right)$ for some $\tau^{\prime} \in B_{n+2}$, so define $p(\sigma)=\sigma^{\prime}$ and $h_{\sigma}=h_{\sigma^{\prime}}^{-1} \cup$ $\left\{\left\langle\inf \left(\omega \backslash \operatorname{ran} h_{\sigma^{\prime}}\right), k_{n-1}\right\rangle\right\}$. In case $\sigma \notin \operatorname{ran} \theta$, define $p(\sigma)=\theta\left(\sigma\left\lceil k_{n-1}^{2}, \sigma\right)\right.$ and $h_{\sigma}=h_{p(\sigma)}^{-1}$.

It remains to verify that conditions 1-5 are satisfied by these definitions of $h$ and $p$ via $\theta$. Note that for $\sigma$ off the $x-y$ branch, $h_{\sigma}=h_{p(\sigma)}^{-1}$ iff $p(\sigma)$ is defined by the second case. Clearly, condition 3 will hold. Observe that if $p(\sigma)=\theta\left(\sigma\left\lceil k_{n-1}^{2}, \sigma\right)\right.$ is defined by the second case then $p(p(\sigma))=p\left(\theta\left(\sigma\left\lceil k_{n-1}^{2}, \sigma\right)\right)=\sigma\left\lceil k_{n-1}^{2}\right.\right.$ must have been defined by the first case. This observation ensures that condition 4 is satisfied.

To verify condition 5 , suppose $\sigma \in B_{n+1}, i \in k_{n-1} \backslash \operatorname{ran} h_{\sigma}, j \in k_{n+1}$, and $h_{\sigma} \neq h_{p(\sigma)}^{-1}$. Here $\sigma=\theta\left(\sigma^{\prime}, \tau^{\prime}\right), p(\sigma)=\sigma^{\prime}$, and $\operatorname{ran} h_{\sigma}=\operatorname{dom} h_{\sigma^{\prime}} \cup\left\{k_{n-1}\right\}$. Now $i \in k_{n-1} \backslash \operatorname{dom} h_{\sigma^{\prime}}$ too, and according to the definition of $\theta\left(\sigma^{\prime}, \tau^{\prime}\right)$

$$
\begin{aligned}
\sigma(i, j) & =\theta\left(\sigma^{\prime}, \tau^{\prime}\right)(i, j) \\
& =d_{i}^{\sigma^{\prime}}(j) \\
& =\inf \left(D \cap\left[\left\langle p\left(\sigma^{\prime}\right)(i, l): l \in k_{n-1}\right\rangle\right]\right)(j) \\
& =\inf \left(D \cap\left[\left\langle p(p(\sigma))(i, l): l \in k_{n-1}\right\rangle\right]\right)(j) .
\end{aligned}
$$

To verify condition 2 , suppose $\sigma \in B_{n}, i \in \operatorname{dom} h_{\sigma}$, and $j \in k_{n-1}$. The first case is when $\sigma=\theta\left(\sigma^{\prime}, \tau^{\prime}\right)$ and $h_{\sigma}=h_{\sigma^{\prime}}^{-1} \cup\left\{\left\langle\inf \left(\omega \backslash \operatorname{ran} h_{\sigma^{\prime}}\right), k_{n-2}\right\rangle\right\}$. Here $i \in \operatorname{ran} h_{\sigma^{\prime}}$ or $i=\inf \left(\omega \backslash \operatorname{ran} h_{\sigma^{\prime}}\right)$. When $i \in \operatorname{ran} h_{\sigma^{\prime}} \subset \operatorname{dom} h_{\sigma}, h_{\sigma}(i) \in \operatorname{dom} h_{\sigma^{\prime}}$, so according to the definition of $\theta\left(\sigma^{\prime}, \tau^{\prime}\right)$,

$$
\begin{aligned}
\sigma\left(h_{\sigma}(i), j\right) & =\theta\left(\sigma^{\prime}, \tau^{\prime}\right)\left(h_{\sigma}(i), j\right) \\
& =\tau^{\prime}\left(h_{\sigma^{\prime}}\left(h_{\sigma}(i)\right), j\right) \\
& =\tau^{\prime}(i, j) \\
& =\sigma^{\prime}(i, j) \\
& =p(\sigma)(i, j) .
\end{aligned}
$$


When $i=\inf \left(\omega \backslash \operatorname{ran} h_{\sigma^{\prime}}\right)$.

$$
\begin{aligned}
\sigma\left(h_{\sigma}\left(\inf \left(\omega \backslash \operatorname{ran} h_{\sigma^{\prime}}\right)\right), j\right) & =\sigma\left(k_{n-2}, j\right) \\
& =\theta\left(\sigma^{\prime}, \tau^{\prime}\right)\left(k_{n-2}, j\right) \\
& =\tau^{\prime}\left(\inf \left(\omega \backslash \operatorname{ran} h_{\sigma^{\prime}}\right), j\right) \\
& =\sigma^{\prime}\left(\inf \left(\omega \backslash \operatorname{ran} h_{\sigma^{\prime}}\right), j\right) \\
& =p(\sigma)\left(\inf \left(\omega \backslash \operatorname{ran} h_{\sigma^{\prime}}\right), j\right) .
\end{aligned}
$$

The second case is when $p(\sigma)=\theta\left(\sigma \nmid k_{n-2}^{2}, \sigma\right)$ and

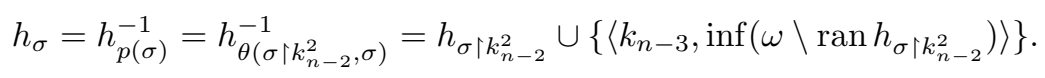

Here $i \in \operatorname{dom} h_{\sigma \nmid k_{n-2}^{2}}$ or $i=k_{n-3}$. According to the definition of $\theta\left(\sigma \uparrow k_{n-2}^{2}, \sigma\right)$, when $i \in \operatorname{dom} h_{\sigma \uparrow k_{n-2}^{2}}$,

$$
\begin{aligned}
p(\sigma)(i, j) & =\theta\left(\sigma\left\lceil k_{n-2}^{2}, \sigma\right)(i, j)\right. \\
& =\sigma\left(h_{\sigma\left\lceil k_{n-2}^{2}\right.}(i), j\right) \\
& =\sigma\left(h_{\sigma}(i), j\right) .
\end{aligned}
$$

When $i=k_{n-3}$,

$$
\begin{aligned}
p(\sigma)\left(k_{n-3}, j\right) & =\theta\left(\sigma\left\lceil k_{n-2}^{2}, \sigma\right)\left(k_{n-3}, j\right)\right. \\
& =\sigma\left(\inf \left(\omega \backslash \operatorname{ran} h_{\sigma\left\lceil k_{n-2}^{2}\right.}\right), j\right) \\
& =\sigma\left(h_{\sigma}\left(k_{n-3}\right), j\right) .
\end{aligned}
$$

To verify condition 1 , suppose $\sigma \in B_{n+1}$. The first case is when $\sigma=\theta\left(\sigma^{\prime}, \tau^{\prime}\right)$ and $p(p(\sigma))=p\left(p\left(\theta\left(\sigma^{\prime}, \tau^{\prime}\right)\right)\right)=p\left(\sigma^{\prime}\right)$. Let $i, j \in k_{n-1}$. According to the definition of $\theta\left(\sigma^{\prime}, \tau^{\prime}\right)$, when $i \in \operatorname{dom} h_{\sigma^{\prime}}$,

$$
\begin{aligned}
\theta\left(\sigma^{\prime}, \tau^{\prime}\right)(i, j) & =\tau^{\prime}\left(h_{\sigma^{\prime}}(i), j\right) \\
& =\sigma^{\prime}\left(h_{\sigma^{\prime}}(i), j\right) \\
& =p\left(\sigma^{\prime}\right)(i, j)
\end{aligned}
$$

because condition 2 has already been verified. When $i \notin \operatorname{dom} h_{\sigma^{\prime}}$,

$$
\begin{aligned}
\theta\left(\sigma^{\prime}, \tau^{\prime}\right)(i, j) & =d_{i}^{\sigma^{\prime}}(j) \\
& =\inf \left(D \cap\left[\left\langle p\left(\sigma^{\prime}\right)(i, l): l \in k_{n-1}\right\rangle\right]\right)(j) \\
& =p\left(\sigma^{\prime}\right)(i, j) .
\end{aligned}
$$

In second case when $p(\sigma)=\theta\left(\sigma \nmid k_{n-1}^{2}, \sigma\right)$, already

$$
p(p(\sigma))=p\left(\theta\left(\sigma\left\lceil k_{n-1}^{2}, \sigma\right)\right)=\sigma\left\lceil k_{n-1}^{2} \subset \sigma .\right.\right.
$$

\section{The generalization}

We extend Lawrence's theorem on first-countable zero-dimensional spaces of countable weight to such spaces of arbitrary weight.

Theorem 2. If $X$ is a first-countable zero-dimensional space then $X^{\omega}$ is homogeneous. 
Proof. Let $X$ be a zero-dimensional first-countable space. $X$ may be identified as a subspace of the Cantor cube $2^{\kappa}$, where $\kappa$ is the weight of $X$. Fix $x, y \in X^{\omega}$. Note that for each $d \in X$ there is a countable subset $S(d)$ of $\kappa$ that determines the topology at $d$. Specifically, $\{a \in X: d \uparrow S \subset a\}, S \in[S(d)]^{<\omega}$ is a neighbourhood basis at $d \in X$. Let $\mathcal{M}$ be a countable elementary submodel containing $x, y, \omega, \kappa, X$ and $\langle S(d): d \in X\rangle$. Note that $x, y \in(X \cap \mathcal{M})^{\omega}$. When $d \in X \cap \mathcal{M}, S(d) \in \mathcal{M}$ by elementarity, thus $S(d) \subset \kappa \cap \mathcal{M}$. Let $\pi$ be the projection $2^{\kappa} \rightarrow 2^{\kappa \cap \mathcal{M}}$. Set $\Pi:\left(2^{\kappa}\right)^{\omega} \rightarrow\left(2^{\kappa \cap \mathcal{M}}\right)^{\omega}$ to be the product of the projections, that is, $\Pi(z)=\langle\pi(z(k))$ : $k \in \omega\rangle$.

Towards applying Theorem 1 to $\pi(X)$, set $D=\pi(X \cap \mathcal{M})$. To see that $D$ is dense in $\pi(X)$, consider a non-empty basic open set of $\pi(X), U_{g}=\{a \in \pi(X): g \subset a\}$, where $g$ is a finite partial function $\kappa \cap \mathcal{M} \rightarrow 2$. All such $g$ are actually in $\mathcal{M}$. $U_{g}$ being non-empty means that $(\exists a \in X) g \subset \pi(a)$. As $\pi(a) \subset a$, by elementarity there is an $a_{\mathcal{M}} \in X \cap \mathcal{M}$ such that $g \subset a_{\mathcal{M}}$. Now $\pi\left(a_{\mathcal{M}}\right) \in U_{g} \cap D$.

If $c \in D$ then there is a unique $a \in X$ such that $\pi(a)=c$. Of course, here $a \in X \cap \mathcal{M}$. In particular, $\pi$ is one-to-one on $\pi^{-1}(D)$. To see this, let $c \in D$. Find $a \in X \cap \mathcal{M}$ with $\pi(a)=c$. Suppose $b \in X$ with $\pi(b)=c$ too. If $a \neq b$ then they can be separated by a neighbourhood of $a$. There must be some $\alpha \in S(a)$ such that $a(\alpha) \neq b(\alpha)$. But $\alpha \in S(a) \subset \kappa \cap \mathcal{M}$, contradicting $\pi(a)=\pi(b)$.

Apply Theorem 1 to find $f$, a continuous translation of $\Pi\left(X^{\omega}\right)=\pi(X)^{\omega}$ modulo $D$ coded by $h$, such that $f(\Pi(x))=\Pi(y)$. In particular, for $z \in X^{\omega}$ and $n \in$ $\operatorname{dom} h_{\Pi(z)}$,

$$
f(\Pi(z))(n)=\Pi(z)\left(h_{\Pi(z)}(n)\right)=\pi\left(z\left(h_{\Pi(z)}(n)\right)\right) .
$$

Lift $f$ to $F: X^{\omega} \rightarrow X^{\omega}$ as follows. Let $z \in X^{\omega}$. For $n \in \operatorname{dom} h_{\Pi(z)}$, define $F(z)(n)=z\left(h_{\Pi(z)}(n)\right)$. Note

$$
\pi(F(z)(n))=\pi\left(z\left(h_{\Pi(z)}(n)\right)\right)=\Pi(z)\left(h_{\Pi(z)}(n)\right)=f(\Pi(z))(n) .
$$

When $n \notin \operatorname{dom} h_{\Pi(z)}, f(\Pi(z))(n) \in \pi(X \cap \mathcal{M})$, so define $F(z)(n)$ as the unique element in $X \cap \mathcal{M}$ such that $\pi(F(z)(n))=f(\Pi(z))(n)$. Observe that $\Pi(F(z))=$ $f(\Pi(z))$.

To prove that $F$ is continuous, it suffices to check that $F$ preserves convergent sequences. Suppose $z_{i} \rightarrow z$ in $X^{\omega}$. Convergence is checked coordinate-wise, so it would suffice that $(\forall n \in \omega) F\left(z_{i}\right)(n) \rightarrow F(z)(n)$. When $n \in \operatorname{dom} h_{\Pi(z)}, F(z)(n)=$ $z\left(h_{\Pi(z)}(n)\right)$ and the continuity of $h$ ensures that eventually $h_{\Pi\left(z_{i}\right)}(n)=h_{\Pi(z)}(n)$. Therefore, eventually

$$
F\left(z_{i}\right)(n)=z_{i}\left(h_{\Pi\left(z_{i}\right)}(n)\right)=z_{i}\left(h_{\Pi(z)}(n)\right) \rightarrow z\left(h_{\Pi(z)}(n)\right)=F(z)(n) .
$$

When $n \notin \operatorname{dom} h_{\Pi(z)}, F(z)(n) \in X \cap \mathcal{M}$. Since the topology at $F(z)(n)$ is determined by $S(F(z)(n)) \subset \kappa \cap \mathcal{M}$, it suffices that $\pi\left(F\left(z_{i}\right)(n)\right) \rightarrow \pi(F(z)(n))$. But already

$$
\pi\left(F\left(z_{i}\right)(n)\right)=f\left(\Pi\left(z_{i}\right)\right)(n) \rightarrow f(\Pi(z))(n)=\pi(F(z)(n)) .
$$

To show that $F$ is one-to-one, let $z, w \in X^{\omega}$ and suppose that $F(z)=F(w)$. Here $f(\Pi(z))=\Pi(F(z))=\Pi(F(w))=f(\Pi(w)) . f$ is one-to-one, therefore $\Pi(z)=\Pi(w)$. When $m \in \operatorname{ran} h_{\Pi(z)}$, there is an $n \in \operatorname{dom} h_{\Pi(z)}$ such that $m=h_{\Pi(z)}(n)=h_{\Pi(w)}(n)$. 
Thus

$$
z(m)=z\left(h_{\Pi(z)}(n)\right)=F(z)(n)=F(w)(n)=w\left(h_{\Pi(w)}(n)\right)=w(m) .
$$

When $m \notin \operatorname{ran} h_{\Pi(z)}, \pi(z(m)) \in D$ and since $\pi(z(m))=\pi(w(m))$, we conclude that $z(m)=w(m)$.

Towards proving that $F$ is surjective, let $w \in X^{\omega}$. Find any $z \in X^{\omega}$ such that $f(\Pi(z))=\Pi(w)$. Define $\tilde{z} \in X^{\omega}$ by

$$
\tilde{z}(m)= \begin{cases}w\left(h_{\Pi(z)}^{-1}(m)\right), & m \in \operatorname{ran} h_{\Pi(z)}, \\ z(m), & m \notin \operatorname{ran} h_{\Pi(z)} .\end{cases}
$$

Note that $\Pi(\tilde{z})=\Pi(z)$. Indeed, when $m \in \operatorname{ran} h_{\Pi(z)}, h_{\Pi(z)}^{-1}(m) \in \operatorname{dom} h_{\Pi(z)}$, so according to the definition of $F(z)$,

$$
\begin{aligned}
\pi(\tilde{z}(m)) & =\pi\left(w\left(h_{\Pi(z)}^{-1}(m)\right)\right) \\
& =\Pi(w)\left(h_{\Pi(z)}^{-1}(m)\right) \\
& =f(\Pi(z))\left(h_{\Pi(z)}^{-1}(m)\right) \\
& =\Pi(F(z))\left(h_{\Pi(z)}^{-1}(m)\right) \\
& =\pi\left(F(z)\left(h_{\Pi(z)}^{-1}(m)\right)\right) \\
& =\pi\left(z\left(h_{\Pi(z)}\left(h_{\Pi(z)}^{-1}(m)\right)\right)\right) \\
& =\pi(z(m))
\end{aligned}
$$

and this is clearly also true for $m \notin \operatorname{ran} h_{\Pi(z)}$. It remains to verify that $F(\tilde{z})=w$. For $n \in \operatorname{dom} h_{\Pi(\tilde{z})}$,

$$
F(\tilde{z})(n)=\tilde{z}\left(h_{\Pi(\tilde{z})}(n)\right)=\tilde{z}\left(h_{\Pi(z)}(n)\right)=w\left(h_{\Pi(z)}^{-1}\left(h_{\Pi(z)}(n)\right)\right)=w(n) .
$$

For $n \notin \operatorname{dom} h_{\Pi(\tilde{z})}, F(\tilde{z})(n)$ is unique such that $\pi(F(\tilde{z})(n))=f(\Pi(\tilde{z}))(n)$. But $\pi(w(n))=\Pi(w)(n)=f(\Pi(z))(n)=f(\Pi(\tilde{z}))(n)$ too.

To conclude that $F$ is a homeomorphism, it remains to show that $F^{-1}$ is continuous. That proof is similar to the proof of continuity of $F$.

The following theorem may be proven by a similar argument.

Theorem 3. If $X$ is zero-dimensional and has a dense subset of points of countable character then $X^{\omega}$ is homogeneous with respect to points of countable character.

\section{REFERENCES}

[1] Gary Gruenhage, New Classic Problems: Homogeneity of $X^{\infty}$, Top. Proc. 15 (1990) 207208

[2] Gary Gruenhage and Hao-Xuan Zhou, Homogeneity of $X^{\omega}$, notes

[3] O.H. Keller, Die homoiomorphie der kompakten konvexen Mengen im Hilbertschen Raum, Math. Ann. 105 (1931) 748-758 MR 41:7258

[4] L. Brian Lawrence, Homogeneity in powers of subspaces of the real line, manuscript, 1994

[5] S.V. Medvedev, Characterizations of h-homogeneous metric spaces, Interim Reports of the Prague Topological Symposium 2 (1987) 19

[6] D.B. Motorov, Homogeneity and $\pi$-networks, Moscow Univ. Math. Bull. 44 (1989) no.4, $45-50$ 
[7] Fons van Engelen, On the homogeneity of infinite products, Top. Proc. 17 (1992) 303-315 MR 95b:54044

[8] Hao-Xuan Zhou, Homogeneity properties and power spaces, Dissertation, Wesleyan University (1993)

Department of Mathematics and Statistics, York University, North York, Ontario, CANADA M3J 1P3

E-mail address: adow@yorku.ca

E-mail address: elliott.pearl@mathstat.yorku.ca 